PFT and Sleep Diagnostics, Mullingar, Ireland.

\title{
Respiratory physiologist training in Ireland
}

Respiratory physiologists are part of a large multidisciplinary team. Their role is to investigate respiratory and sleep disorders through various diagnostic tools and methodologies. In Ireland, respiratory physiologists complete a 4-year Bachelor of Science (BSc) degree in clinical measurement science.

Previously, for more than 30 years (and up to 2004), a part-time certificate programme in medical physics and physiological measurement was offered to allow people to work in respiratory and sleep diagnostics. In line with the movements in many education and training initiatives in the health and social care sector within Ireland, clinical measurement science was validated as a full-time honours (Hons) degree programme.

\section{What is a BSc Hons in clinical measurement science?}

The BSc in clinical measurement science programme is designed to educate and train clinical measurement physiologists who perform diagnostic tests in the areas of cardiac physiology, neurophysiology, respiratory physiology or vascular physiology.

\section{Structure of the clinical measurement degree programme in Ireland}

The degree extends over 4 years of full-time study, and consists of lectures, tutorials, assignments, laboratory work, clinical placements and project work.

Years 1 and 2 provide students with a foundation in science subjects with the introduction of each of the four clinical measurement science specialties on offer within the degree programme: cardiology, neurophysiology, respiratory and vascular.

Year 3 is largely hospital based. Following a series of lectures in college, students spend 24 weeks on placement in rotation through three of the four clinical disciplines listed above.
Year 4 of the programme involves a mixture of taught material, a hospital clinical placement of 12-14 weeks and a research project. In the final year, students study two of the three disciplines undertaken in year 3 with a clinical placement in one of these disciplines (this being the student's major discipline). Prior to the commencement of year 4, students will decide which discipline in which to major, and those who choose respiratory will thus qualify as respiratory physiologists.

\section{Respiratory physiologist post-qualification}

Once qualified and finished the 4-year degree programme in clinical measurement science, a respiratory physiologist's role is to investigate respiratory and sleep disorders. Respiratory physiologist graduates may also undertake the Irish Association of Respiratory Scientists National Assessment in Lung Function when they have been working full time for 1 year.

Many different patients are sent for pulmonary function (respiratory) tests (PFTs). Most patients are undergoing investigations for respiratory problems but many patients are referred for different reasons. Specialists in cardiology, oncology, rheumatology, neurology, dermatology, endocrinology, haematology and infectious disease often request PFTs. In addition to these specialist medical referrals, we provide services to surgical specialities including cardiothoracic surgery and heart transplantation, pre-operative assessments, and general practitioner referrals.

\section{Respiratory investigations}

Respiratory physiologists are responsible for delivering the highest standards in physiological measurements to our patients in both respiratory and sleep medicine. The following respiratory investigations are available for the screening and evaluation of respiratory disease, and are performed by the Respiratory Physiologist.
Cite as: Flood E. Respiratory physiologist training in Ireland. Breathe 2017; 13: 255-257. 
The first three (spirometry, and measurement of static lung volumes and single-breathe diffusing capacity of the lung for carbon monoxide (DıCO)) are usually performed together during one testing session to form the baseline PFT result.

\section{Spirometry}

Spirometry is invaluable as a screening test of general respiratory health in the same way that blood pressure provides important information about general cardiovascular health. It is a physiological test that measures how an individual inhales or exhales volumes of air as a function of time. The primary signals measured in spirometry are volume and flow.

\section{Static lung volumes: gas methods}

The gas methods measure three lung volumes, functional residual capacity, residual volume and total lung capacity, which are used to classify restrictive impairments, confirm small airway obstruction and quantify the degree of air trapping in overt airway obstruction. In contrast to the relative simplicity of spirometric volumes, a variety of techniques have been developed for the measurement of absolute lung volumes. These include gas dilution techniques (nitrogen or helium dilution) and body plethysmography

\section{Single-breath $D$ Lco}

Measurement of single-breathe DLCO provides information about the transfer of oxygen between the alveoli and the pulmonary-capillary blood. Respiratory conditions such as pulmonary fibrosis, sarcoidosis, restrictive lung disease and pulmonary emboli that affect the interstitial areas of the lungs require assessment of gas exchange to support the diagnosis and monitor these conditions over time.

\section{Respiratory muscle strength}

The measurement of maximal inspiratory pressure via the mouth or sniff nasal inspiratory pressure via the nose, are important tests used to determine inspiratory muscle strength. The primary usefulness of these measurements is in the diagnosis of patients with neuromuscular disease or injuries involving the inspiratory muscles. They are also useful for evaluating patients whose strength of inspiration is reduced secondary to hyperinflation, severe chest wall deformities, or drugs.

\section{Hypoxic challenge test: fitness-to-fly assessment}

Air travel is a common mode of travel for millions of people worldwide and for the vast majority it is safe; however, for patients with compromised lung function due to a variety of respiratory diseases, it may not be so. Cabin altitudes of up to $2400 \mathrm{~m}$ result in progressive decreases in cabin oxygen levels to $\sim 15 \%$ that may induce hypoxia in patients with lung disease, which could lead to respiratory arrest in severe cases. A fitness-to-fly test is therefore a valuable tool for the physician issuing recommendations to patients with lung disease wishing to undertake air travel, particularly long-haul flights.

\section{Skin allergy tests}

Skin allergy tests are performed on the surface skin of the forearm of a patient, producing immediate reactions that can detect causative factors in atopic allergy. Atopic diseases include hay fever, perennial rhinitis, eczema and asthma. Allergens commonly tested for include house dust mite, selections of pollens, animal hairs, moulds and fungi.

\section{Cardiopulmonary exercise test}

Cardiopulmonary exercise testing (CPET) provides a global assessment of the integrative exercise responses involving the pulmonary, cardiovascular, haematopoietic, neurophysiological and skeletal muscle systems. It is therefore a valuable tool in stressing the organs involved and determining what abnormalities exist. This relatively noninvasive, dynamic physiological overview permits the evaluation of both submaximal and peak exercise responses, providing a consultant with relevant information for clinical decision making. CPET is increasingly being used for the evaluation of undiagnosed exercise intolerance or unexplained breathlessness and exercise-related symptoms, for the determination of functional capacity and impairment as part of the pre-assessment for heart/lung transplant, and/or before major thoracic surgery.

\section{Exhaled nitric oxide}

Nitric oxide is present in exhaled breath of patients and is a useful marker of airway inflammation. A nitric oxide analyser is a high sensitivity detector for measuring nitric oxide, and is based on gasphase chemiluminescent reaction between nitric oxide and ozone. The exhaled nitric oxide (eNO) measurements are in parts per billion. Increased levels of eNO indicate airway inflammation.

\section{Bronchial challenge testing}

Bronchial challenge testing is used to identify and characterise airway hyperresponsiveness by provoking airway changes in susceptible patients. Bronchial challenge tests are also sometimes used to screen individuals who may be at risk from environmental or occupational exposure 
to toxins. This challenge test uses a stimulant to provoke airway changes in susceptible patients. A number of stimuli can be used, such as exercise, occupational exposures, and chemical aerosolised substances such as methacholine/histamine, or a dry-powder formula like mannitol. Respiratory physiologists must be highly trained to perform these types of tests.

\section{Sleep investigations}

The following sleep investigations are available for the screening and evaluation of sleep disorders and are performed by the respiratory physiologist. Investigations can be diagnostic or therapeutic.

- Diagnostic: investigation of sleep disturbances associated with a range of conditions. Studies range in complexity from assessment of overnight respiratory parameters to polysomnographic studies investigating respiratory and neurological sleep disturbances.

- Therapeutic: overnight studies used to determine optimal treatment of sleep disorders, in particular obstructive sleep apnoea syndrome (OSAS), and to monitor efficacy of treatment.

Sleep investigations and screening carried out in the sleep laboratory include the following/

\section{Cardiorespiratory sleep study}

A limited cardiorespiratory sleep study records respiratory events only; the sleep/wake stages are not assessed. Ventilation (airflow), and respiratory and oxygen saturation are recorded. Body position, snore events, bilateral leg electromyography (EMG) and heart rate via a three-lead ECG are often also recorded depending on the equipment available. The computerised automated analysis is manually edited according to national and international standardised guidelines, by trained and experienced respiratory physiologists, after the study has been completed, to ensure accurate and reliable results are available for clinical interpretation.

\section{Polysomnography}

Polysomnography (PSG) assesses both sleep and respiration on a multichannel recorder. PSG is considered the gold-standard test but it is very time consuming and is reserved for patients without obvious clinical signs of OSAS (i.e. investigation of narcolepsy, period leg movement disorder, etc.) or in patients where a limited study did not provide a definitive diagnosis. Along with the respiratory sensors listed under the limited sleep study, other physiological signals are measured such as electroencephalography for brain activity, electrooculography to detect eye movement and EMG on the chin. This multichannel recording is then manually scored by highly trained respiratory physiologists.

\section{Continuous positive airway pressure therapy}

A continuous positive airway pressure (CPAP) machine is an small electrical device that takes in air from the room, lightly pressurises it and delivers it through a flexible tube to a special mask attached to the patient's nose or nose and mouth (full-face mask). This air is under positive pressure and acts as a splint to keep the airway open, thus eliminating obstructive apnoeas. This treatment gives immediate relief to OSAS sufferers by providing uninterrupted breathing and sleep patterns.

\section{CPAP/automatic positive airway pressure compliance monitoring}

Many sleep patients are requested to bring their CPAP/automatic positive airway pressure devices and mask system with them each time they attend the sleep clinic for review. A microchip in these devices records various details including the amount of time the patient had been wearing the mask and how many nights the patient is on treatment, as the treatment is only effective while the patients use the device during sleep on a continuous basis. The respiratory physiologist downloads the compliance data from the CPAP unit, usually via a personal computer, and the relevant information is then recorded in the patient healthcare record for review by the medical team.

\section{Day-to-day work of the respiratory physiologist}

A typical day working as a respiratory physiologist can vary significantly from department to department and from hospital to hospital within Ireland. Many PFT and sleep departments perform most of the above investigations; however, unfortunately, sometimes due to budgets, staffing or equipment, some departments may not be able to perform the full range described here.

In the area of respiratory diagnostics, there is an increasing demand on our services due to increasing prevalence of conditions such as chronic obstructive pulmonary disease, asthma and sleep apnoea, and hence, many respiratory physiologists are much busier now than ever before.

\section{Conflict of interest}

None declared. 